$\left\{\begin{array}{l}\text { Revista Integración } \\ \text { Escuela de Matemáticas } \\ \text { Universidad Industrial de Santander } \\ \text { Vol. 33, No. 2, 2015, pág. 173-189 }\end{array}\right.$

DOI: http://dx.doi.org/10.18273/revint.v33n2-2015007

\title{
Skew PBW Extensions of Baer, quasi-Baer, p.p. and p.q.-rings
}

\author{
ARMAndo REYES* \\ Universidad Nacional de Colombia, Departamento de Matemáticas, Bogotá, Colombia.
}

\begin{abstract}
The aim of this paper is to study skew Poincaré-Birkhoff-Witt extensions of Baer, quasi-Baer, p.p. and p.q.-Baer rings. Using a notion of rigidness, we prove that these properties are stable over this kind of extensions.

Keywords: Baer, quasi-Baer, p.p. and p.q.-Baer rings, skew PoincaréBirkhoff-Witt extensions.
\end{abstract}

MSC2010: 16E50, 16S36, 16D25.

\section{Extensiones PBW torcidas de anillos de Baer, quasi-Baer, p.p. y p.q-Baer}

Resumen. El propósito de este artículo es estudiar las extensiones torcidas de Poincaré-Birkhoff-Witt de anillos de Baer, quasi-Baer, p.p. y p.q.-Baer. Utilizando una noción de rigidez, probamos que estas propiedades son estables para esta clase de extensiones.

Palabras clave: Anillos Baer, quasi-Baer, p.p, p.q.-Baer, extensiones torcidas de Poincaré-Birkhoff-Witt.

\section{Introduction}

Kaplansky [15] defined a ring $B$ as a Baer (resp. quasi-Baer, which was defined by Clark [6]) ring if the right annihilator of every nonempty subset (resp. ideal) of $B$ is generated by an idempotent. Another generalization of Baer rings are the p.p.-rings. A ring $B$ is called right (resp. left) p.p if the right (resp. left) annihilator of each element of $B$ is generated by an idempotent (or equivalently, rings in which each principal right (resp. left) ideal is projective). Birkenmeier et al. [4] define a ring to be called a right (resp. left) principally quasi-Baer (or simply right (resp. left) p.q-Baer) ring if the right annihilator of each principal right (resp. left) ideal of $B$ is generated by an idempotent.

*E-mail: mareyesv@unal.edu.co

Received: 25 September 2015, Accepted: 17 November 2015.

To cite this article: A. Reyes, Skew PBW Extensions of Bear, quasi-Baer, p.p. and p.q.-rings, Rev. Integr

Temas Mat. 33 (2015), No. 2, 173-189. 
Commutative and noncommutative rings Baer, quasi-Baer, p.p.-rings, and right p.q.Baer have been investigated in the literature. For instance, polynomial extensions in the commutative case were studied in [1],[3], and Ore extensions $B[x ; \alpha, \delta]$ of injective type, i.e., when $\alpha$ is injective, of all this kind of rings can be found in several works (cf. [4],[5],[8],[10],[11],[13],[14], and others). Some of these works consider the case $\delta=0$ and $\alpha$ an automorphism, or the case where $\alpha$ is the identity. Nevertheless, it is important to say that the Baerness and quasi-Baerness of a ring $B$ and an Ore extension $B[x ; \sigma, \delta]$ of $B$ does not depend on each other. More exactly, there are examples which show that there exists a Baer ring $B$ but the Ore extension $B[x ; \sigma, \delta]$ is not right p.q.Baer; similarly, there exist Ore extensions $B[x ; \sigma, \delta]$ which are quasi-Baer, but $B$ is not quasi-Baer (see [13] for more details). With this in mind, a natural question for a given class of Baer, quasi-Baer, p.p.-rings, and right p.q.-Baer, is the behavior with respect to skew Poincaré-Birkhoff-Witt (PBW for short) extensions (introduced in [7]), which are more general than Ore extensions of injective type. More exactly, it has been shown that skew PBW extensions contain various well-known groups of algebras such as some types of Auslander-Gorenstein rings, some skew Calabi-Yau algebras, quantum polynomials, some quantum universal enveloping algebras, etc. (see [17] or [23]). In fact, these extensions include several algebras which can not be expressed as Ore extensions (universal enveloping algebras of finite Lie algebras, diffusion algebras, and others, see Section 4). This shows the necessity to have more general results in a theory of Baerness and quasi-Baerness for several noncommutative rings. Therefore, this paper contains a first approach about Baer, quasi-Baer, p.p. and p.q.-rings with the purpose of establishing necessary and sufficient conditions to guarantee that these properties are stable over skew PBW extensions. Since we use a notion of rigidness (see Definition 3.2), we generalize the results presented in [13] and [21] using techniques fairly standard and following the same path as other text on the subject (see Theorems 3.9, 3.10,3.12, and 3.13, which are the important results of this paper). In this way, we continue the task of studying ring and module theoretical properties of skew PBW extensions (cf. [17],[18],[23],[24], and others).

\section{Definitions and elementary properties}

In this section we recall the definition of skew PBW extensions and present some key properties of these rings.

Definition 2.1 ([7], Definition 1). Let $R$ and $A$ be rings. We say that $A$ is a skew $P B W$ extension of $R$ (also called a $\sigma-P B W$ extension of $R$ ) if the following conditions hold:

(i) $R \subseteq A$;

(ii) there exist elements $x_{1}, \ldots, x_{n} \in A$ such that $A$ is a left free $R$-module, with basis the basic elements $\operatorname{Mon}(A):=\left\{x^{\alpha}=x_{1}^{\alpha_{1}} \cdots x_{n}^{\alpha_{n}} \mid \alpha=\left(\alpha_{1}, \ldots, \alpha_{n}\right) \in \mathbb{N}^{n}\right\}$.

(iii) For each $1 \leq i \leq n$ and any $r \in R \backslash\{0\}$, there exists an element $c_{i, r} \in R \backslash\{0\}$ such that $x_{i} r-c_{i, r} x_{i} \in R$.

(iv) For any elements $1 \leq i, j \leq n$ there exists $c_{i, j} \in R \backslash\{0\}$ such that $x_{j} x_{i}-c_{i, j} x_{i} x_{j} \in$ $R+R x_{1}+\cdots+R x_{n}$. 
Under these conditions we will write $A:=\sigma(R)\left\langle x_{1}, \ldots, x_{n}\right\rangle$.

Remark 2.2 ([7], Remark 2). (i) Since $\operatorname{Mon}(A)$ is a left $R$-basis of $A$, the elements $c_{i, r}$ and $c_{i, j}$ in Definition 2.1 are unique; (ii) In Definition 2.1 (iv), $c_{i, i}=1$. This follows from $x_{i}^{2}-c_{i, i} x_{i}^{2}=s_{0}+s_{1} x_{1}+\cdots+s_{n} x_{n}$, with $s_{i} \in R$, which implies $1-c_{i, i}=0=s_{i}$.

Proposition 2.3 ([7], Proposition 3). Let $A$ be a skew PBW extension of $R$. For each $1 \leq i \leq n$, there exists an injective endomorphism $\sigma_{i}: R \rightarrow R$ and an $\sigma_{i}$-derivation $\delta_{i}: R \rightarrow R$ such that $x_{i} r=\sigma_{i}(r) x_{i}+\delta_{i}(r)$, for each $r \in R$.

Remark 2.4. Following the notation of Proposition 2.3, we write $\Sigma:=\left\{\sigma_{1}, \ldots, \sigma_{n}\right\}$, and $\Delta:=\left\{\delta_{1}, \ldots, \delta_{n}\right\}$, that is, $\Delta$ is the family of $\Sigma$-derivations in $A$.

A particular case of skew PBW extension is considered when derivations $\delta_{i}$ are zero for every $i$. Another case is presented when all endomorphisms $\sigma_{i}$ are isomorphisms. These observations are formulated in the next definition.

Definition 2.5 ([7], Definition 4). Let $A$ be a skew PBW extension of $R$.

(a) $A$ is called quasi-commutative if the conditions (iii) and (iv) in Definition 2.1 are replaced by (iii'): for each $1 \leq i \leq n$ and all $r \in R \backslash\{0\}$ there exists $c_{i, r} \in R \backslash\{0\}$ such that $x_{i} r=c_{i, r} x_{i}$; (iv'): for any $1 \leq i, j \leq n$ there exists $c_{i, j} \in R \backslash\{0\}$ such that $x_{j} x_{i}=c_{i, j} x_{i} x_{j}$.

(b) $A$ is called bijective if $\sigma_{i}$ is bijective for each $1 \leq i \leq n$, and $c_{i, j}$ is invertible for any $1 \leq i<j \leq n$.

Example 2.6. The class of skew $P B W$ extensions contains various well-known groups of algebras such as some types of Auslander-Gorenstein rings, some skew Calabi-Yau algebras, quantum polynomials, some quantum universal enveloping algebras, etc. A detailed list of examples of skew PBW extensions is presented in [17],[23] or [24].

Definition 2.7 ([7], Definition 6). Let $A$ be a skew PBW extension of $R$ with endomorphisms $\sigma_{i}, 1 \leq i \leq n$, as in Proposition 2.3 .

(i) For $\alpha=\left(\alpha_{1}, \ldots, \alpha_{n}\right) \in \mathbb{N}^{n}, \sigma^{\alpha}:=\sigma_{1}^{\alpha_{1}} \cdots \sigma_{n}^{\alpha_{n}},|\alpha|:=\alpha_{1}+\cdots+\alpha_{n}$. If $\beta=$ $\left(\beta_{1}, \ldots, \beta_{n}\right) \in \mathbb{N}^{n} ;$ then $\alpha+\beta:=\left(\alpha_{1}+\beta_{1}, \ldots, \alpha_{n}+\beta_{n}\right)$.

(ii) For $X=x^{\alpha} \in \operatorname{Mon}(A), \exp (X):=\alpha$ and $\operatorname{deg}(X):=|\alpha|$. The symbol $\succeq$ will denote a total order defined on $\operatorname{Mon}(A)$ (a total order on $\mathbb{N}_{0}^{n}$ ). For an element $x^{\alpha} \in \operatorname{Mon}(A), \exp \left(x^{\alpha}\right):=\alpha \in \mathbb{N}_{0}^{n}$. If $x^{\alpha} \succeq x^{\beta}$ but $x^{\alpha} \neq x^{\beta}$, we write $x^{\alpha} \succ x^{\beta}$. Every element $f \in A$ can be expressed uniquely as $f=a_{0}+a_{1} X_{1}+\cdots+a_{m} X_{m}$, with $a_{i} \in R \backslash\{0\}$, and $X_{m} \succ \cdots \succ X_{1}$. With this notation, we define $\operatorname{lm}(f):=X_{m}$, the leading monomial of $f ; \operatorname{lc}(f):=a_{m}$, the leading coefficient of $f ; \operatorname{lt}(f):=a_{m} X_{m}$, the leading term of $f ; \exp (f):=\exp \left(X_{m}\right)$, the order of $f$; and $E(f):=\left\{\exp \left(X_{i}\right) \mid 1 \leq\right.$ $i \leq t\}$. Note that $\operatorname{deg}(f):=\max \left\{\operatorname{deg}\left(X_{i}\right)\right\}_{i=1}^{t}$. Finally, if $f=0$, then $\operatorname{lm}(0):=0$, $\operatorname{lc}(0):=0, \operatorname{lt}(0):=0$. We also consider $X \succ 0$ for any $X \in \operatorname{Mon}(A)$. For a detailed description of monomial orders in skew PBW extensions, see [7], Section 3.

Skew PBW extensions can be characterized in the following way.

Vol. 33, No. 2, 2015] 
Theorem 2.8 ([7], Theorem 7). Let $A$ be a polynomial ring over $R$ with respect to $\left\{x_{1}, \ldots, x_{n}\right\} . A$ is a skew $P B W$ extension of $R$ if and only if the following conditions are satisfied:

(i) for each $x^{\alpha} \in \operatorname{Mon}(A)$ and every $0 \neq r \in R$, there exist unique elements $r_{\alpha}:=$ $\sigma^{\alpha}(r) \in R \backslash\{0\}, p_{\alpha, r} \in A$, such that $x^{\alpha} r=r_{\alpha} x^{\alpha}+p_{\alpha, r}$, where $p_{\alpha, r}=0$ or $\operatorname{deg}\left(p_{\alpha, r}\right)<|\alpha|$ if $p_{\alpha, r} \neq 0$. If $r$ is left invertible, so is $r_{\alpha}$.

(ii) For each $x^{\alpha}, x^{\beta} \in \operatorname{Mon}(A)$ there exist unique elements $c_{\alpha, \beta} \in R$ and $p_{\alpha, \beta} \in A$ such that $x^{\alpha} x^{\beta}=c_{\alpha, \beta} x^{\alpha+\beta}+p_{\alpha, \beta}$, where $c_{\alpha, \beta}$ is left invertible, $p_{\alpha, \beta}=0$ or $\operatorname{deg}\left(p_{\alpha, \beta}\right)<$ $|\alpha+\beta|$ if $p_{\alpha, \beta} \neq 0$.

In Proposition 2.9 and Remark 2.10 we will look more closely at the form of the polynomials $p_{\alpha, r}$ and $p_{\alpha, \beta}$ in Theorem 2.8. We start establishing an expression for the product $x^{\alpha} r=x_{1}^{\alpha_{1}} \cdots x_{n}^{\alpha_{n}} r$, with $\alpha:=\left(\alpha_{1}, \ldots, \alpha_{n}\right) \in \mathbb{N}_{0}^{n}$ and $r \in R$.

Proposition 2.9. We have the equality

$$
\begin{aligned}
x^{\alpha} r & =x_{1}^{\alpha_{1}} x_{2}^{\alpha_{2}} \cdots x_{n-1}^{\alpha_{n-1}} x_{n}^{\alpha_{n}} r=x_{1}^{\alpha_{1}} \cdots x_{n-1}^{\alpha_{n-1}}\left(\sum_{j=1}^{\alpha_{n}} x_{n}^{\alpha_{n}-j} \delta_{n}\left(\sigma_{n}^{j-1}(r)\right) x_{n}^{j-1}\right) \\
& +x_{1}^{\alpha_{1}} \cdots x_{n-2}^{\alpha_{n}-2}\left(\sum_{j=1}^{\alpha_{n-1}} x_{n-1}^{\alpha_{n-1}-j} \delta_{n-1}\left(\sigma_{n-1}^{j-1}\left(\sigma_{n}^{\alpha_{n}}(r)\right)\right) x_{n-1}^{j-1}\right) x_{n}^{\alpha_{n}} \\
& +x_{1}^{\alpha_{1}} \cdots x_{n-3}^{\alpha_{n-3}}\left(\sum_{j=1}^{\alpha_{n-2}} x_{n-2}^{\alpha_{n-2}-j} \delta_{n-2}\left(\sigma_{n-2}^{j-1}\left(\sigma_{n-1}^{\alpha_{n-1}}\left(\sigma_{n}^{\alpha_{n}}(r)\right)\right)\right) x_{n-2}^{j-1}\right) x_{n-1}^{\alpha_{n-1}} x_{n}^{\alpha_{n}} \\
& +\cdots+x_{1}^{\alpha_{1}}\left(\sum_{j=1}^{\alpha_{2}} x_{2}^{\alpha_{2}-j} \delta_{2}\left(\sigma_{2}^{j-1}\left(\sigma_{3}^{\alpha_{3}}\left(\sigma_{4}^{\alpha_{4}}\left(\cdots\left(\sigma_{n}^{\alpha_{n}}(r)\right)\right)\right)\right)\right) x_{2}^{j-1}\right) x_{3}^{\alpha_{3}} x_{4}^{\alpha_{4}} \cdots x_{n-1}^{\alpha_{n-1}} x_{n}^{\alpha_{n}} \\
& +\sigma_{1}^{\alpha_{1}}\left(\sigma_{2}^{\alpha_{2}}\left(\cdots\left(\sigma_{n}^{\alpha_{n}}(r)\right)\right)\right) x_{1}^{\alpha_{1}} \cdots x_{n}^{\alpha_{n}}, \quad \sigma_{j}^{0}:=\mathrm{id}_{R} \quad \text { for } 1 \leq j \leq n .
\end{aligned}
$$

Proof. Induction on the number of variables. Let us see the case $n=1$. Let $x_{n}$ be a variable and $\alpha_{n}$ an element of $\mathbb{N}_{0}$. The idea is to show that

$$
x_{n}^{\alpha_{n}} r=\sigma_{n}^{\alpha_{n}}(r) x_{n}^{\alpha_{n}}+\sum_{j=1}^{\alpha_{n}} x_{n}^{\alpha_{n}-j} \delta_{n}\left(\sigma_{n}^{j-1}(r)\right) x_{n}^{j-1}, \quad \sigma_{n}^{0}:=\operatorname{id}_{R} .
$$

If $\alpha_{n}=0$ or 1 , the result is clear. Suppose the assertion is true for $\alpha_{n}$. Then

$$
\begin{aligned}
x_{n}^{\alpha_{n}+1} r & =x_{n} x_{n}^{\alpha_{n}} r=x_{n}\left(\sigma_{n}^{\alpha_{n}}(r) x_{n}^{\alpha_{n}}+\sum_{j=1}^{\alpha_{n}} x_{n}^{\alpha_{n}-j} \delta_{n}\left(\sigma_{n}^{j-1}(r)\right) x_{n}^{j-1}\right) \\
& =x_{n} \sigma_{n}^{\alpha_{n}}(r) x_{n}^{\alpha_{n}}+x_{n}\left(\sum_{j=1}^{\alpha_{n}} x_{n}^{\alpha_{n}-j} \delta_{n}\left(\sigma_{n}^{j-1}(r)\right) x_{n}^{j-1}\right) \\
& =\left(\sigma_{n}^{\alpha_{n}+1}(r) x_{n}+\delta_{n}\left(\sigma_{n}^{\alpha_{n}}(r)\right)\right) x_{n}^{\alpha_{n}}+\sum_{j=1}^{\alpha_{n}} x_{n}^{\alpha_{n}+1-j} \delta_{n}\left(\sigma_{n}^{j-1}(r)\right) x_{n}^{j-1} \\
& =\sigma_{n}^{\alpha_{n}+1}(r) x_{n}^{\alpha_{n}+1}+\sum_{j=1}^{\alpha_{n}+1} x_{n}^{\alpha_{n}+1-j} \delta_{n}\left(\sigma_{n}^{j-1}(r)\right) x_{n}^{j-1},
\end{aligned}
$$

[Revista Integración 
which proves the case $n=1$.

Suppose that the assertion is true for $n$. Let us see the situation when we have $n+1$ variables.

$$
\begin{aligned}
x_{1}^{\alpha_{1}} \cdots x_{n+1}^{\alpha_{n+1}} r=x_{1}^{\alpha_{1}} \cdots x_{n}^{\alpha_{n}}\left(\sigma_{n+1}^{\alpha_{n+1}}(r) x_{n+1}^{\alpha_{n+1}}+\sum_{j=1}^{\alpha_{n+1}} x_{n+1}^{\alpha_{n+1}-j} \delta_{n+1}\left(\sigma_{n+1}^{j-1}(r)\right) x_{n+1}^{j-1}\right) \\
=x_{1}^{\alpha_{1}} \cdots x_{n}^{\alpha_{n}} \sigma_{n+1}^{\alpha_{n+1}}(r) x_{n+1}^{\alpha_{n}}+x_{1}^{\alpha_{1}} \cdots x_{n}^{\alpha_{n}} \sum_{j=1}^{\alpha_{n+1}} x_{n+1}^{\alpha_{n+1}-j} \delta_{n+1}\left(\sigma_{n+1}^{j-1}(r)\right) x_{n+1}^{j-1} \\
=\left[x_{1}^{\alpha_{1}} \cdots x_{n-1}^{\alpha_{n-1}}\left(\sum_{j=1}^{\alpha_{n}} x_{n}^{\alpha_{n}-j} \delta_{n}\left(\sigma_{n}^{j-1}\left(\sigma_{n+1}^{\alpha_{n+1}}(r)\right)\right) x_{n}^{j-1}\right)\right. \\
\quad+x_{1}^{\alpha_{1}} \cdots x_{n-2}^{\alpha_{n-2}}\left(\sum_{j=1}^{\alpha_{n-1}} x_{n-1}^{\alpha_{n-1}-j} \delta_{n-1}\left(\sigma_{n-1}^{j-1}\left(\sigma_{n}^{\alpha_{n}}\left(\sigma_{n+1}^{\alpha_{n+1}}(r)\right)\right)\right) x_{n-1}^{j-1}\right) x_{n}^{\alpha_{n}} \\
\quad+x_{1}^{\alpha_{1}} \cdots x_{n-3}^{\alpha_{n-3}}\left(\sum_{j=1}^{\alpha_{n-2}} x_{n-2}^{\alpha_{n-2}-j} \delta_{n-2}\left(\sigma_{n-2}^{j-1}\left(\sigma_{n-1}^{\alpha_{n-1}}\left(\sigma_{n}^{\alpha_{n}}\left(\sigma_{n+1}^{\alpha_{n+1}}(r)\right)\right)\right)\right) x_{n-2}^{j-1}\right) x_{n-1}^{\alpha_{n-1}} x_{n}^{\alpha_{n}} \\
\quad+\cdots+x_{1}^{\alpha_{1}}\left(\sum_{j=1}^{\alpha_{2}} x_{2}^{\alpha_{2}-j} \delta_{2}\left(\sigma_{2}^{j-1}\left(\sigma_{3}^{\alpha_{3}}\left(\cdots\left(\sigma_{n}^{\alpha_{n}}\left(\sigma_{n+1}^{\alpha_{n+1}}(r)\right)\right)\right)\right)\right) x_{2}^{j-1}\right) x_{3}^{\alpha_{3}} \cdots x_{n}^{\alpha_{n}} \\
\left.\quad+\sigma_{1}^{\alpha_{1}}\left(\cdots\left(\sigma_{n}^{\alpha_{n}}\left(\sigma_{n+1}^{\alpha_{n+1}}(r)\right)\right)\right) x_{1}^{\alpha_{1}} \cdots x_{n}^{\alpha_{n}}\right] x_{n+1}^{\alpha_{n+1}} \\
\quad+x_{1}^{\alpha_{1}} \cdots x_{n}^{\alpha_{n}}\left(\sum_{j=1}^{\alpha_{n+1}} x_{n+1}^{\alpha_{n+1}-j} \delta_{n+1}\left(\sigma_{n+1}^{j-1}(r)\right) x_{n+1}^{j-1}\right) .
\end{aligned}
$$

Equivalently,

$$
\begin{aligned}
& x_{1}^{\alpha_{1}} \cdots x_{n}^{\alpha_{n}} x_{n+1}^{\alpha_{n+1}} r=x_{1}^{\alpha_{1}} \cdots x_{n}^{\alpha_{n}}\left(\sum_{j=1}^{\alpha_{n+1}} x_{n+1}^{\alpha_{n+1}-j} \delta_{n+1}\left(\sigma_{n+1}^{j-1}(r)\right) x_{n+1}^{j-1}\right) \\
& \quad+x_{1}^{\alpha_{1}} \cdots x_{n-1}^{\alpha_{n-1}}\left(\sum_{j=1}^{\alpha_{n}} x_{n}^{\alpha_{n}-j} \delta_{n}\left(\sigma_{n}^{j-1}\left(\sigma_{n+1}^{\alpha_{n+1}}(r)\right)\right) x_{n}^{j-1}\right) x_{n+1}^{\alpha_{n+1}} \\
& \quad+x_{1}^{\alpha_{1}} \cdots x_{n-2}^{\alpha_{n-2}}\left(\sum_{j=1}^{\alpha_{n-1}} x_{n-1}^{\alpha_{n-1}-j} \delta_{n-1}\left(\sigma_{n-1}^{j-1}\left(\sigma_{n}^{\alpha_{n}}\left(\sigma_{n+1}^{\alpha_{n+1}}(r)\right)\right)\right) x_{n-1}^{j-1}\right) x_{n}^{\alpha_{n}} x_{n+1}^{\alpha_{n+1}} \\
& \quad+\cdots+x_{1}^{\alpha_{1}}\left(\sum_{j=1}^{\alpha_{2}} x_{2}^{\alpha_{2}-j} \delta_{2}\left(\sigma_{2}^{j-1}\left(\sigma_{3}^{\alpha_{3}} \cdots\left(\sigma_{n+1}^{\alpha_{n+1}}(r)\right)\right)\right) x_{2}^{j-1}\right) x_{3}^{\alpha_{3}} \cdots x_{n}^{\alpha_{n}} x_{n+1}^{\alpha_{n+1}} \\
& \quad+\sigma_{1}^{\alpha_{1}}\left(\cdots\left(\sigma_{n+1}^{\alpha_{n+1}}(r)\right)\right) x_{1}^{\alpha_{1}} \cdots x_{n}^{\alpha_{n}} x_{n+1}^{\alpha_{n+1}} .
\end{aligned}
$$

Remark 2.10. (i) By (1) we know that

$$
\begin{aligned}
x_{n}^{\alpha_{n}} r & =\sigma_{n}^{\alpha_{n}}(r) x_{n}^{\alpha_{n}}+x_{n}^{\alpha_{n}-1} \delta_{n}(r)+x_{n}^{\alpha_{n}-2} \delta_{n}\left(\sigma_{n}(r)\right) x_{n}+x_{n}^{\alpha_{n}-3} \delta_{n}\left(\sigma_{n}^{2}(r)\right) x_{n}^{2} \\
& +\cdots+x_{n} \delta_{n}\left(\sigma_{n}^{\alpha_{n}-2}(r)\right) x_{n}^{\alpha_{n}-2}+\delta_{n}\left(\sigma_{n}^{\alpha_{n}-1}(r)\right) x_{n}^{\alpha_{n}-1}, \quad \sigma_{n}^{0}:=\operatorname{id}_{R} .
\end{aligned}
$$

Vol. 33, No. 2, 2015] 
Note that

$$
\begin{aligned}
p_{\alpha_{n}, r}= & x_{n}^{\alpha_{n}-1} \delta_{n}(r)+x_{n}^{\alpha_{n}-2} \delta_{n}\left(\sigma_{n}(r)\right) x_{n}+x_{n}^{\alpha_{n}-3} \delta_{n}\left(\sigma_{n}^{2}(r)\right) x_{n}^{2} \\
& +\cdots+x_{n} \delta_{n}\left(\sigma_{n}^{\alpha_{n}-2}(r)\right) x_{n}^{\alpha_{n}-2}+\delta_{n}\left(\sigma_{n}^{\alpha_{n}-1}(r)\right) x_{n}^{\alpha_{n}-1},
\end{aligned}
$$

where $p_{\alpha_{n}, r}=0$ or $\operatorname{deg}\left(p_{\alpha_{n}, r}\right)<\alpha_{n}$ if $p_{\alpha_{n}, r} \neq 0$ (Theorem 2.8 (i)). It is clear that $\exp \left(p_{\alpha_{n}, r}\right) \prec \alpha_{n}$. Again, using (1) in every term of the product $x_{n}^{\alpha_{n}} r$ above, we obtain

$$
\begin{aligned}
x_{n}^{\alpha_{n}} r & =\sigma_{n}^{\alpha_{n}}(r) x_{n}^{\alpha_{n}}+\sigma_{n}^{\alpha_{n}-1}\left(\delta_{n}(r)\right) x_{n}^{\alpha_{n}-1}+\sum_{j=1}^{\alpha_{n}-1} x_{n}^{\alpha_{n}-1-j} \delta_{n}\left(\sigma_{n}^{j-1}\left(\delta_{n}(r)\right)\right) x_{n}^{j-1} \\
& +\left[\sigma_{n}^{\alpha_{n}-2}\left(\delta_{n}\left(\sigma_{n}(r)\right)\right) x_{n}^{\alpha_{n}-2}+\sum_{j=1}^{\alpha_{n}-2} x_{n}^{\alpha_{n}-2-j} \delta_{n}\left(\sigma_{n}^{j-1}\left(\delta_{n}\left(\sigma_{n}(r)\right)\right)\right) x_{n}^{j-1}\right] x_{n} \\
& +\left[\sigma_{n}^{\alpha_{n}-3}\left(\delta_{n}\left(\sigma_{n}^{2}(r)\right)\right) x_{n}^{\alpha_{n}-3}+\sum_{j=1}^{\alpha_{n}-3} x_{n}^{\alpha_{n}-3-j} \delta_{n}\left(\sigma_{n}^{j-1}\left(\delta_{n}\left(\sigma_{n}^{2}(r)\right)\right)\right) x_{n}^{j-1}\right] x_{n}^{2} \\
& +\cdots+\left[\sigma_{n}\left(\delta_{n}\left(\sigma_{n}^{\alpha_{n}-2}(r)\right)\right) x_{n}+\delta_{n}\left(\delta_{n}\left(\sigma_{n}^{\alpha_{n}-2}(r)\right)\right)\right] x_{n}^{\alpha_{n}-2}+\delta_{n}\left(\sigma_{n}^{\alpha_{n}-1}(r)\right) x_{n}^{\alpha_{n}-1},
\end{aligned}
$$

which shows that

$$
\operatorname{lc}\left(p_{\alpha_{n}, r}\right)=\sum_{p=1}^{\alpha_{n}} \sigma_{n}^{\alpha_{n}-p}\left(\delta_{n}\left(\sigma_{n}^{p-1}(r)\right)\right) .
$$

In this way, we can see that $\operatorname{lc}\left(p_{\alpha_{n}, r}\right)$ involves elements obtained evaluating $\sigma_{n}$ and $\delta_{n}$ in the element $r$ of $R$. Now, as an illustration, let us see the case of two and three variables with the idea to establish a general fact about the coefficients of the polynomials $p_{\alpha, r}$ and $p_{\alpha, \beta}$ in Theorem 2.8 .

(ii) Consider the product $x_{n-1}^{\alpha_{n-1}} x_{n}^{\alpha_{n}} r$ :

$$
\begin{aligned}
x_{n-1}^{\alpha_{n-1}} x_{n}^{\alpha_{n}} r & =x_{n-1}^{\alpha_{n-1}}\left(\sigma_{n}^{\alpha_{n}}(r) x_{n}^{\alpha_{n}}+p_{\alpha_{n}, r}\right) \\
& =x_{n-1}^{\alpha_{n-1}} \sigma_{n}^{\alpha_{n}}(r) x_{n}^{\alpha_{n}}+x_{n-1}^{\alpha_{n-1}} p_{\alpha_{n}, r} \\
& =\left[\sigma_{n-1}^{\alpha_{n-1}}\left(\sigma_{n}^{\alpha_{n}}(r)\right) x_{n-1}^{\alpha_{n-1}}+p_{\alpha_{n-1}, \sigma_{n}^{\alpha_{n}}(r)}\right] x_{n}^{\alpha_{n}}+x_{n-1}^{\alpha_{n-1}} p_{\alpha_{n}, r},
\end{aligned}
$$

where $p_{\alpha_{n-1}, \sigma_{n}^{\alpha_{n}}(r)}=0$, or $\operatorname{deg}\left(p_{\alpha_{n-1}, \sigma_{n}^{\alpha_{n}}(r)}\right)<\alpha_{n-1}$, if $p_{\alpha_{n-1}, \sigma_{n}^{\alpha_{n}}(r)} \neq 0$. Hence,

$$
x_{n-1}^{\alpha_{n-1}} x_{n}^{\alpha_{n}} r=\sigma_{n-1}^{\alpha_{n-1}}\left(\sigma_{n}^{\alpha_{n}}(r)\right) x_{n-1}^{\alpha_{n-1}} x_{n}^{\alpha_{n}}+p_{\alpha_{n-1}, \sigma_{n}^{\alpha_{n}}(r)} x_{n}^{\alpha_{n}}+x_{n-1}^{\alpha_{n-1}} p_{\alpha_{n}, r},
$$

with relations $\exp \left(p_{\alpha_{n-1}, \sigma_{n}^{\alpha_{n}}(r)} x_{n}^{\alpha_{n}}\right)=\left(\operatorname{deg}\left(p_{\alpha_{n-1}, \sigma_{n}^{\alpha_{n}}(r)}\right), \alpha_{n}\right) \prec\left(\alpha_{n-1}, \alpha_{n}\right)$, $\exp \left(x_{n-1}^{\alpha_{n-1}} p_{\alpha_{n}, r}\right)=\left(\alpha_{n-1}, \operatorname{deg}\left(p_{\alpha_{n}, r}\right)\right) \prec\left(\alpha_{n-1}, \alpha_{n}\right)$, and both degrees $\operatorname{deg}\left(p_{\alpha_{n-1}, \sigma_{n}^{\alpha_{n}}(r)} x_{n}^{\alpha_{n}}\right)$ and $\operatorname{deg}\left(x_{n-1}^{\alpha_{n-1}} p_{\alpha_{n}, r}\right)$ less than $\alpha_{n-1}+\alpha_{n}$. By (2) we have

$$
\operatorname{lc}\left(p_{\alpha_{n-1}, \sigma_{n}^{\alpha_{n}}(r)}\right)=\sum_{p=1}^{\alpha_{n-1}} \sigma_{n-1}^{\alpha_{n-1}-p}\left(\delta_{n-1}\left(\sigma_{n-1}^{p-1}\left(\sigma_{n}^{\alpha_{n}}(r)\right)\right)\right) .
$$

Note that

$$
\operatorname{lc}\left(p_{\alpha_{n-1}, \sigma_{n}^{\alpha_{n}}(r)} x_{n}^{\alpha_{n}}\right)=\operatorname{lc}\left(p_{\alpha_{n-1}, \sigma_{n}^{\alpha_{n}}(r)}\right),
$$


and

$$
\operatorname{lc}\left(x_{n-1}^{\alpha_{n-1}} p_{\alpha_{n}, r}\right)=\sigma_{n-1}^{\alpha_{n-1}}\left(\sum_{p=1}^{\alpha_{n}} \sigma_{n}^{\alpha_{n}-p}\left(\delta_{n}\left(\sigma_{n}^{p-1}(r)\right)\right)\right)=\sum_{p=1}^{\alpha_{n}} \sigma_{n-1}^{\alpha_{n-1}}\left(\sigma_{n}^{\alpha_{n}-p}\left(\delta_{n}\left(\sigma_{n}^{p-1}(r)\right)\right)\right.
$$

In this way (3) can be expressed as

$$
\begin{aligned}
x_{n-1}^{\alpha_{n-1}} x_{n}^{\alpha_{n}} r & =\sigma_{n-1}^{\alpha_{n-1}}\left(\sigma_{n}^{\alpha_{n}}(r)\right) x_{n-1}^{\alpha_{n-1}} x_{n}^{\alpha_{n}} \\
& +\left[\sum_{p=1}^{\alpha_{n-1}} \sigma_{n-1}^{\alpha_{n-1}-p}\left(\delta_{n-1}\left(\sigma_{n-1}^{p-1}\left(\sigma_{n}^{\alpha_{n}}(r)\right)\right)\right)\right] x_{n-1}^{\operatorname{deg}\left(p_{\alpha_{n-1}, \sigma_{n}^{\alpha}(r)}\right)} x_{n}^{\alpha_{n}} \\
& +\left[\sum_{p=1}^{\alpha_{n}} \sigma_{n-1}^{\alpha_{n-1}}\left(\sigma_{n}^{\alpha_{n}-p}\left(\delta_{n}\left(\sigma_{n}^{p-1}(r)\right)\right)\right)\right] x_{n-1}^{\alpha_{n-1}} x_{n}^{\operatorname{deg}\left(p_{\alpha_{n}, r}\right)} \\
& + \text { other terms of degree less than } \operatorname{deg}\left(p_{\alpha_{n-1}, \sigma_{n}^{\alpha_{n}}(r)}\right)+\alpha_{n} \\
& + \text { other terms of degree less than } \alpha_{n-1}+\operatorname{deg}\left(p_{\alpha_{n}, r}\right) .
\end{aligned}
$$

(iii) Let us see a final example considering the case with three variables, that is,

$$
\begin{aligned}
& x_{n-2}^{\alpha_{n-2}} x_{n-1}^{\alpha_{n-1}} x_{n}^{\alpha_{n}} r=x_{n-2}^{\alpha_{n-2}}\left[\sigma_{n-1}^{\alpha_{n-1}}\left(\sigma_{n}^{\alpha_{n}}(r)\right) x_{n-1}^{\alpha_{n-1}} x_{n}^{\alpha_{n}}+p_{\alpha_{n-1}, \sigma_{n}^{\alpha_{n}}(r)} x_{n}^{\alpha_{n}}+x_{n-1}^{\alpha_{n-1}} p_{\alpha_{n}, r}\right] \\
& =x_{n-2}^{\alpha_{n-2}} \sigma_{n-1}^{\alpha_{n-1}}\left(\sigma_{n}^{\alpha_{n}}(r)\right) x_{n-1}^{\alpha_{n-1}} x_{n}^{\alpha_{n}}+x_{n-2}^{\alpha_{n-2}} p_{\alpha_{n-1}, \sigma_{n}^{\alpha_{n}}(r)} x_{n}^{\alpha_{n}} \\
& +x_{n-2}^{\alpha_{n-2}} x_{n-1}^{\alpha_{n-1}} p_{\alpha_{n}, r}
\end{aligned}
$$

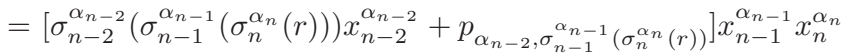

$$
\begin{aligned}
& +x_{n-2}^{\alpha_{n-2}} p_{\alpha_{n-1}, \sigma_{n}^{\alpha_{n}}(r)} x_{n}^{\alpha_{n}}+x_{n-2}^{\alpha_{n-2}} x_{n-1}^{\alpha_{n-1}} p_{\alpha_{n}, r} \text {, }
\end{aligned}
$$

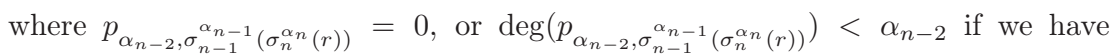

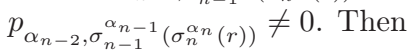

$$
\begin{aligned}
& x_{n-2}^{\alpha_{n-2}} x_{n-1}^{\alpha_{n}} x_{n}^{\alpha_{n}} r=\sigma_{n-2}^{\alpha_{n-2}}\left(\sigma_{n-1}^{\alpha_{n-1}}\left(\sigma_{n}^{\alpha_{n}}(r)\right)\right) x_{n-2}^{\alpha_{n-2}} x_{n-1}^{\alpha_{n-1}} x_{n}^{\alpha_{n}}+p_{\alpha_{n-2}, \sigma_{n-1}^{\alpha_{n-1}}\left(\sigma_{n}^{\alpha_{n}}(r)\right)} x_{n-1}^{\alpha_{n-1}} x_{n}^{\alpha} \\
& +x_{n-2}^{\alpha_{n-2}} p_{\alpha_{n-1}, \sigma_{n}^{\alpha_{n}}(r)} x_{n}^{\alpha_{n}}+x_{n-2}^{\alpha_{n-2}} x_{n-1}^{\alpha_{n-1}} p_{\alpha_{n}, r},
\end{aligned}
$$

where

$$
\begin{aligned}
\exp \left(p_{\alpha_{n-2}, \sigma_{n-1}^{\alpha_{n-1}}\left(\sigma_{n}^{\alpha_{n}}(r)\right)} x_{n-1}^{\alpha_{n-1}} x_{n}^{\alpha_{n}}\right) & =\left(\operatorname{deg}\left(p_{\alpha_{n-2}, \sigma_{n-1}^{\alpha_{n-1}}\left(\sigma_{n}^{\alpha_{n}}(r)\right)}\right), \alpha_{n-1}, \alpha_{n}\right) \\
\exp \left(x_{n-2}^{\alpha_{n-2}} p_{\alpha_{n-1}, \sigma_{n}^{\alpha_{n}}(r)} x_{n}^{\alpha_{n}}\right) & =\left(\alpha_{n-2}, \operatorname{deg}\left(p_{\alpha_{n-1}, \sigma_{n}^{\alpha_{n}}(r)}, \alpha_{n}\right)\right. \\
\exp \left(x_{n-2}^{\alpha_{n-2}} x_{n-1}^{\alpha_{n-1}} p_{\alpha_{n}, r}\right) & =\left(\alpha_{n-2}, \alpha_{n-1}, \operatorname{deg}\left(p_{\alpha_{n}, r}\right)\right),
\end{aligned}
$$

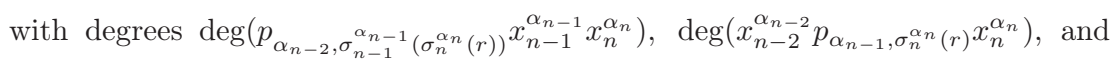
$\operatorname{deg}\left(x_{n-2}^{\alpha_{n-2}} x_{n-1}^{\alpha_{n-1}} p_{\alpha_{n}, r}\right)$ less than $\alpha_{n-2}+\alpha_{n-1}+\alpha_{n}$. It is clear that

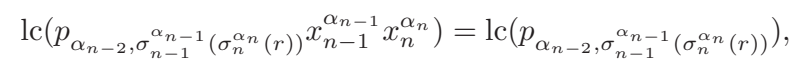


and by (2) and (4),

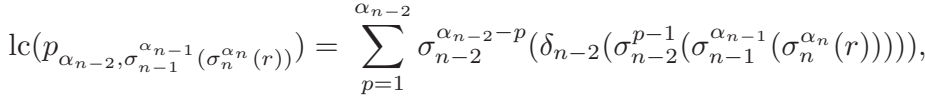

$$
\begin{aligned}
& \operatorname{lc}\left(x_{n-2}^{\alpha_{n-2}} p_{\alpha_{n-1}, \sigma_{n}^{\alpha_{n}}(r)} x_{n}^{\alpha_{n}}\right)=\sigma_{n-2}^{\alpha_{n-2}}\left(\sum_{p=1}^{\alpha_{n-1}} \sigma_{n-1}^{\alpha_{n-1}-p}\left(\delta_{n-1}\left(\sigma_{n-1}^{p-1}\left(\sigma_{n}^{\alpha_{n}}(r)\right)\right)\right)\right) \\
& =\sum_{p=1}^{\alpha_{n-1}} \sigma_{n-2}^{\alpha_{n-2}}\left(\sigma_{n-1}^{\alpha_{n-1}-p}\left(\delta_{n-1}\left(\sigma_{n-1}^{p-1}\left(\sigma_{n}^{\alpha_{n}}(r)\right)\right)\right)\right), \\
& \operatorname{lc}\left(x_{n-2}^{\alpha_{n-2}} x_{n-1}^{\alpha_{n-1}} p_{\alpha_{n}, r}\right)=\sigma_{n-2}^{\alpha_{n-2}}\left(\sigma_{n-1}^{\alpha_{n-1}}\left(\sum_{p=1}^{\alpha_{n}} \sigma_{n}^{\alpha_{n}-p}\left(\delta_{n}\left(\sigma_{n}^{p-1}(r)\right)\right)\right)\right) \\
& =\sum_{p=1}^{\alpha_{n}} \sigma_{n-2}^{\alpha_{n-2}}\left(\sigma_{n-1}^{\alpha_{n-1}}\left(\sigma_{n}^{\alpha_{n}-p}\left(\delta_{n}\left(\sigma_{n}^{p-1}(r)\right)\right)\right)\right) \text {. }
\end{aligned}
$$

Then the term $x_{n-2}^{\alpha_{n-2}} x_{n-1}^{\alpha_{n-1}} x_{n}^{\alpha_{n}} r$ can be written as

$$
\begin{aligned}
& \sigma_{n-2}^{\alpha_{n-2}}\left(\sigma_{n-1}^{\alpha_{n-1}}\left(\sigma_{n}^{\alpha_{n}}(r)\right)\right) x_{n-2}^{\alpha_{n-2}} x_{n-1}^{\alpha_{n-1}} x_{n}^{\alpha_{n}} \\
+ & {\left[\sum_{p=1}^{\alpha_{n-2}} \sigma_{n-2}^{\alpha_{n-2}-p}\left(\delta_{n-2}\left(\sigma_{n-2}^{p-1}\left(\sigma_{n-1}^{\alpha_{n-1}}\left(\sigma_{n}^{\alpha_{n}}(r)\right)\right)\right)\right) x_{n-2}^{\operatorname{deg}\left(p_{\alpha_{n-2}, \sigma_{n-1} \alpha_{n-1}\left(\sigma_{n}^{\alpha}(r)\right)}\right)}\right] x_{n-1}^{\alpha_{n-1}} x_{n}^{\alpha_{n}} } \\
+ & {\left[\sum_{p=1}^{\alpha_{n-1}} \sigma_{n-2}^{\alpha_{n-2}}\left(\sigma_{n-1}^{\alpha_{n-1}-p}\left(\delta_{n-1}\left(\sigma_{n-1}^{p-1}\left(\sigma_{n}^{\alpha_{n}}(r)\right)\right)\right)\right)\right] x_{n-2}^{\alpha_{n-2}} x_{n-1}^{\operatorname{deg}\left(p_{\alpha_{n-1}, \sigma_{n}^{\alpha}(r)}\right)} x_{n}^{\alpha_{n}} } \\
+ & {\left[\sum_{p=1}^{\alpha_{n}} \sigma_{n-2}^{\alpha_{n-2}}\left(\sigma_{n-1}^{\alpha_{n-1}}\left(\sigma_{n}^{\alpha_{n}-p}\left(\delta_{n}\left(\sigma_{n}^{p-1}(r)\right)\right)\right)\right)\right] x_{n-2}^{\alpha_{n-2}} x_{n-1}^{\alpha_{n-1}} x_{n}^{\operatorname{deg}\left(p_{\alpha_{n}, r}\right)} } \\
+ & \text { other terms of degree less than } \operatorname{deg}\left(p_{\alpha_{n-2}, \sigma_{n-1}^{\alpha_{n-1}}\left(\sigma_{n}^{\left.\alpha_{n}(r)\right)}\right)}\right)+\alpha_{n-1}+\alpha_{n} \\
+ & \text { other terms of degree less than } \alpha_{n-2}+\operatorname{deg}\left(p_{\left.\alpha_{n-1}, \sigma_{n}^{\alpha_{n}(r)}\right)}\right)+\alpha_{n} \\
+ & \text { other terms of degree less than } \alpha_{n-2}+\alpha_{n-1}+\operatorname{deg}\left(p_{\alpha_{n}, r}\right) .
\end{aligned}
$$

Continuing in this way we can see that for $n$ variables we have

$$
\begin{aligned}
x_{1}^{\alpha_{1}} x_{2}^{\alpha_{2}} \cdots x_{n-1}^{\alpha_{n-1}} x_{n}^{\alpha_{n}} r & =\sigma_{1}^{\alpha_{1}}\left(\cdots\left(\sigma_{n}^{\alpha_{n}}(r)\right)\right) x_{1}^{\alpha_{1}} x_{2}^{\alpha_{2}} \cdots x_{n}^{\alpha_{n}} \\
& +p_{\alpha_{1}, \sigma_{2}^{\alpha_{2}}\left(\cdots\left(\sigma_{n}^{\alpha_{n}}(r)\right)\right)} x_{2}^{\alpha_{2}} \cdots x_{n}^{\alpha_{n}} \\
& +x_{1}^{\alpha_{1}} p_{\alpha_{2}, \sigma_{3}^{\alpha_{3}}\left(\cdots\left(\sigma_{n}^{\alpha_{n}}(r)\right)\right)} x_{3}^{\alpha_{3}} \cdots x_{n}^{\alpha_{n}} \\
& +x_{1}^{\alpha_{1}} x_{2}^{\alpha_{2}} p_{\alpha_{3}, \sigma_{4}^{\alpha_{4}}\left(\cdots\left(\sigma_{n}^{\alpha_{n}}(r)\right)\right)} x_{4}^{\alpha_{4}} \cdots x_{n}^{\alpha_{n}} \\
& +\cdots+x_{1}^{\alpha_{1}} x_{2}^{\alpha_{2}} \cdots x_{n-2}^{\alpha_{n}-2} p_{\alpha_{n-1}, \sigma_{n}^{\alpha_{n}}(r)} x_{n}^{\alpha_{n}} \\
& +x_{1}^{\alpha_{1}} \cdots x_{n-1}^{\alpha_{n-1}} p_{\alpha_{n}, r} .
\end{aligned}
$$


Considering the leading coefficients of $x_{1}^{\alpha_{1}} \cdots x_{n}^{\alpha_{n}} r$ we can write this term as

$$
\begin{aligned}
& =\sigma_{1}^{\alpha_{1}}\left(\cdots\left(\sigma_{n}^{\alpha_{n}}(r)\right)\right) x_{1}^{\alpha_{1}} \cdots x_{n}^{\alpha_{n}} \\
& \left.+\left[\sum_{p=1}^{\alpha_{1}} \sigma_{1}^{\alpha_{1}-p}\left(\delta_{1}\left(\sigma_{1}^{p-1}\left(\sigma_{2}^{\alpha_{2}}\left(\sigma_{3}^{\alpha_{3}}\left(\cdots\left(\sigma_{n}^{\alpha_{n}}(r)\right)\right)\right)\right)\right)\right)\right] x_{1}^{\operatorname{deg}(p} \alpha_{\alpha_{1}, \sigma_{2}^{\alpha_{2}}\left(\cdots\left(\sigma_{n}^{\alpha_{n}}(r)\right)\right)}\right) x_{2}^{\alpha_{2}} \cdots x_{n}^{\alpha_{n}}
\end{aligned}
$$

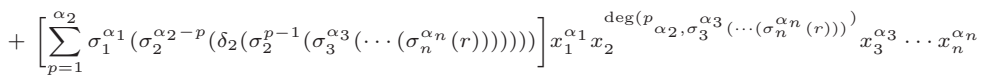

$$
\begin{aligned}
& +\left[\sum_{p=1}^{\alpha_{3}} \sigma_{1}^{\alpha_{1}}\left(\sigma_{2}^{\alpha_{2}}\left(\sigma_{3}^{\alpha_{3}-p}\left(\delta_{3}\left(\sigma_{3}^{p-1}\left(\sigma_{4}^{\alpha_{4}}\left(\cdots\left(\sigma_{n}^{\alpha_{n}}(r)\right)\right)\right)\right)\right)\right)\right)\right] x_{1}^{\alpha_{1}} x_{2}^{\alpha_{2}} x_{3}^{\operatorname{deg}(p}{ }_{\alpha_{3}, \sigma_{4} \alpha_{4}\left(\cdots\left(\sigma_{n}^{\alpha_{n}}(r)\right)\right)}{ }^{\alpha_{4}} x_{4}^{\alpha_{4}} \cdots x_{n}^{\alpha_{n}} \\
& +\cdots+\left[\sum_{p=1}^{\alpha_{n}-1} \sigma_{1}^{\alpha_{1}}\left(\cdots\left(\sigma_{n-2}^{\alpha_{n-2}}\left(\sigma_{n-1}^{\alpha_{n-1}-p}\left(\delta_{n-1}\left(\sigma_{n-1}^{p-1}\left(\sigma_{n}^{\alpha_{n}}(r)\right)\right)\right)\right)\right)\right)\right] x_{1}^{\alpha_{1}} \cdots x_{n-2}^{\alpha_{n-2}} x_{n-1}^{\operatorname{deg}(p}{ }_{\alpha_{n-1}, \sigma_{n}^{\alpha_{n}}(r)}{ }^{\alpha_{2}} x_{n}^{\alpha_{n}} \\
& +\left[\sum_{p=1}^{\alpha_{n}} \sigma_{1}^{\alpha_{1}}\left(\cdots\left(\sigma_{n-1}^{\alpha_{n-1}}\left(\sigma_{n}^{\alpha_{n}-p}\left(\delta_{n}\left(\sigma_{n}^{p-1}(r)\right)\right)\right)\right)\right)\right] x_{1}^{\alpha_{1}} \cdots x_{n-1}^{\alpha_{n-1}} x_{n}^{\operatorname{deg}\left(p_{\alpha_{n}, r}\right)} \\
& + \text { other terms of degree less than } \operatorname{deg}\left(p_{\alpha_{1}, \sigma_{2}^{\alpha_{2}}\left(\cdots\left(\sigma_{n}^{\alpha_{n}}(r)\right)\right)}\right)+\alpha_{2}+\cdots+\alpha_{n} \\
& + \text { other terms of degree less than } \alpha_{1}+\operatorname{deg}\left(p_{\alpha_{2}, \sigma_{3}^{\alpha_{3}}\left(\cdots\left(\sigma_{n}^{\alpha_{n}}(r)\right)\right)}\right)+\alpha_{3}+\cdots+\alpha_{n} \\
& + \text { other terms of degree less than } \alpha_{1}+\alpha_{2}+\operatorname{deg}\left(p_{\alpha_{3}, \sigma_{4}^{\alpha}}^{\alpha_{4}}\left(\cdots\left(\sigma_{n}^{\alpha_{n}}(r)\right)\right)+\alpha_{4}+\cdots+\alpha_{n}\right. \\
& + \text { other terms of degree less than } \alpha_{1}+\cdots+\alpha_{n-2}+\operatorname{deg}\left(p_{\alpha_{n-1}, \sigma_{n}^{\alpha_{n}(r)}}\right)+\alpha_{n} \\
& + \text { other terms of degree less than } \alpha_{1}+\cdots+\alpha_{n-1}+\operatorname{deg}\left(p_{\alpha_{n}, r}\right) \text {. }
\end{aligned}
$$

Therefore we can see that the polynomials $p_{\alpha_{1}, \sigma_{2}^{\alpha_{2}}\left(\cdots\left(\sigma_{n}^{\alpha_{n}}(r)\right)\right)}, p_{\alpha_{2}, \sigma_{3}^{\alpha_{3}}\left(\cdots\left(\sigma_{n}^{\alpha_{n}}(r)\right)\right)}$, $p_{\alpha_{3}, \sigma_{4}^{\alpha_{4}}\left(\cdots\left(\sigma_{n}^{\alpha_{n}}(r)\right)\right)}, \ldots, p_{\alpha_{n-1}, \sigma_{n}^{\alpha_{n}}(r)}$, and $p_{\alpha_{n}, r}$ in the expression above for the term $x_{1}^{\alpha_{1}} x_{2}^{\alpha_{2}} \cdots x_{n-1}^{\alpha_{n-1}} x_{n}^{\alpha_{n}} r$, involve elements obtained evaluating $\sigma$ 's and $\delta$ 's in the element $r$ of $R$.

(iv) Consider the product $a_{i} X_{i} b_{j} Y_{j}$. If $X_{i}:=x_{1}^{\alpha i 1} \cdots x_{n}^{\alpha_{i n}}$ and $Y_{j}:=x_{1}^{\beta_{j 1}} \cdots x_{n}^{\beta_{j n}}$. Then

$$
\begin{aligned}
a_{i} X_{i} b_{j} Y_{j} & =a_{i} \sigma^{\alpha_{i}}\left(b_{j}\right) x^{\alpha_{i}} x^{\beta_{j}}+a_{i} p_{\alpha_{i 1}, \sigma_{i 2}^{\alpha_{i 2}}\left(\cdots\left(\sigma_{i n}^{\alpha_{i n}}(b)\right)\right)} x_{2}^{\alpha_{i 2}} \cdots x_{n}^{\alpha_{i n}} x^{\beta_{j}} \\
& +a_{i} x_{1}^{\alpha_{i 1}} p_{\alpha_{i 2}, \sigma_{3}^{\alpha_{i 3}}\left(\cdots\left(\sigma_{i n}^{\alpha_{i n}}(b)\right)\right)} x_{3}^{\alpha_{i 3}} \cdots x_{n}^{\alpha_{i n}} x^{\beta_{j}} \\
& +a_{i} x_{1}^{\alpha_{i 1}} x_{2}^{\alpha_{i 2}} p_{\alpha_{i 3}, \sigma_{i 4}^{\alpha_{i 4}}\left(\cdots\left(\sigma_{i n}^{\alpha_{i n}}(b)\right)\right)} x_{4}^{\alpha_{i 4}} \cdots x_{n}^{\alpha_{i n}} x^{\beta_{j}} \\
& +\cdots+a_{i} x_{1}^{\alpha_{i 1}} x_{2}^{\alpha_{i 2}} \cdots x_{i(n-2)}^{\alpha_{i(n-2)}} p_{\alpha_{i(n-1)}, \sigma_{i n}^{\alpha_{i n}}(b)} x_{n}^{\alpha_{i n}} x^{\beta_{j}} \\
& +a_{i} x_{1}^{\alpha_{i 1}} \cdots x_{i(n-1)}^{\alpha_{i(n-1)}} p_{\alpha_{i n}, b} x^{\beta_{j}}
\end{aligned}
$$

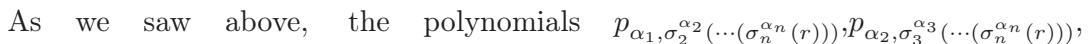
$p_{\alpha_{3}, \sigma_{4}^{\alpha_{4}}\left(\cdots\left(\sigma_{n}^{\alpha_{n}}(r)\right)\right)}, \ldots, p_{\alpha_{n-1}, \sigma_{n}^{\alpha_{n}}(r)}$, and $p_{\alpha_{n}, r}$, involve elements of $R$ obtained evaluating $\sigma_{j}$ and $\delta_{j}$ in the element $r$ of $R$. So, when we compute every summand of $a_{i} X_{i} b_{j} Y_{j}$ we obtain products of the coefficient $a_{i}$ with several evaluations of $b_{j}$ in $\sigma$ 's and $\delta$ 's depending of the coordinates of $\alpha_{i}$.

\section{3. $\quad$ L-Rigid, Baer, quasi-Baer, p.p. and p.q.-rings}

There are important examples of Baer rings which motivate the study of this notion. For instance, von Neumann algebras (e.g., the algebra of all bounded operators on a Hilbert

Vol. 33, No. 2, 2015] 
space), the commutative $C^{*}$-algebra $C(T)$ of continuous complex valued functions on a Stonian space, and others, are remarkable examples in several contexts of mathematics. Concerning ring theory, it is important to say that the class of Baer rings does not contain the class of prime rings (these rings are quasi-Baer [5]) and is not closed under extensions to matrix rings or triangular matrix rings. In the case of Ore extensions, the Baerness and quasi-Baerness of a ring $B$ do not inherite the Ore extension of $B$. More exactly, there are examples which show that there exists a Baer ring $B$ but the Ore extension $B[x ; \sigma, \delta]$ is not right p.q.-Baer; similarly, there exist Ore extensions $B[x ; \sigma, \delta]$ which are quasi-Baer, but $B$ is not quasi-Baer. In general, the Baerness of $B$ and $B[x ; \sigma, \delta]$ does not depend on each other (see [13], Examples 8, 9 and 10). Since Ore extensions of injective type are particular examples of skew PBW extensions, the concepts of Baer, quasi-Baer, and p.p. and p.q. are interesting for the ring theoretical study of skew PBW extensions. Hence, in this section we generalize the results presented in [13] with the purpose of establishing necessary and sufficient conditions to guarantee that these concepts are stable under skew PBW extensions.

We recall some well-known facts. For a nonempty subset $D$ of a ring $B$, we write $r_{B}(D)=\{c \in B \mid D c=0\}$ and $l_{B}(D)=\{c \in B \mid c D=0\}$, which are called the right annihilator of $D$ in $B$ and the left annihilator of $D$ in $B$, respectively. We recall that a ring $B$ is reduced if $B$ has no nonzero nilpotent elements, and a $\operatorname{ring} B$ is called abelian if every idempotent is central. Reduced rings are abelian and also semiprime (that is, its prime radical is trivial), see [3].

We start with the following important result about reduced rings.

Lemma 3.1 ([13], Lemma 1). Let B be a reduced ring. Then the following statements are equivalent:

(i) $B$ is a right p.p.-ring;

(ii) $B$ is a p.p.-ring;

(iii) $B$ is a right p.q.-Baer ring;

(iv) $B$ is a p.q.-Baer ring.

For a ring $B$ with a ring endomorphism $\sigma: B \rightarrow B$, an $\sigma$-derivation $\delta: B \rightarrow B$, considering the Ore extension $B[x ; \sigma, \delta]$, Krempa in [16] defined $\sigma$ as a rigid endomorphism if $b \sigma(b)=0$ implies $b=0$ for $b \in B$. Krempa called $B \sigma$-rigid if there exists a rigid endomorphism $\sigma$ of $B$. Since Ore extensions of injective type are particular examples of skew PBW extensions, we present the following definition with the purpose of studying the notion of rigidness for these extensions.

Definition 3.2. Let $B$ be a ring and $\Sigma$ a family of endomorphisms of $B$. $\Sigma$ is called a rigid endomorphisms family if $r \sigma^{\alpha}(r)=0$ implies $r=0$ for every $r \in B$ and $\alpha \in \mathbb{N}^{n}$. A ring $B$ is called to be $\Sigma$-rigid if there exists a rigid endomorphisms family $\Sigma$ of $B$.

Note that if $\Sigma$ is a rigid endomorphisms family, then every element $\sigma_{i} \in \Sigma$ is a monomorphism. In fact, $\Sigma$-rigid rings are reduced rings: if $B$ is a $\Sigma$-rigid ring and $r^{2}=0$ for $r \in B$, then $0=r \sigma^{\alpha}\left(r^{2}\right) \sigma^{\alpha}\left(\sigma^{\alpha}(r)\right)=r \sigma^{\alpha}(r) \sigma^{\alpha}(r) \sigma^{\alpha}\left(\sigma^{\alpha}(r)\right)=r \sigma^{\alpha}(r) \sigma^{\alpha}\left(r \sigma^{\alpha}(r)\right)$, i.e., 
$r \sigma^{\alpha}(r)=0$ and so $r=0$, that is, $B$ is reduced (note that there exists an endomorphism of a reduced ring which is not a rigid endomorphism, see [13], Example 9). With this in mind, we consider the family of injective endomorphisms $\Sigma$ and the family $\Delta$ of $\Sigma$-derivations in a skew PBW extension $A$ of a ring $R$ (see Remark 2.4).

Lemma 3.3. Let $B$ be an $\Sigma$-rigid ring and $a, b \in B$. Then:

(i) If $a b=0$ then $a \sigma^{\alpha}(b)=\sigma^{\alpha}(a) b=0$ for $\alpha \in \mathbb{N}^{n}$.

(ii) If $a b=0$ then $a \delta^{\beta}(b)=\delta^{\beta}(a) b=0$ for $\beta \in \mathbb{N}^{n}$.

(iii) If $a b=0$ then $a \sigma^{\alpha}\left(\delta^{\beta}(b)\right)=a \delta^{\beta}\left(\sigma^{\alpha}(b)\right)=0$ for every $\alpha, \beta \in \mathbb{N}^{n}$.

(iv) If $a \sigma^{\theta}(b)=\sigma^{\theta}(a) b=0$ for some $\theta \in \mathbb{N}^{n}$, then $a b=0$.

Proof. We follow the ideas presented in [13], Lemma 4.

(i) It is enough to show that if $a b=0$, then $a \sigma_{i}(b)=\sigma_{i}(a) b=0$ for every $1 \leq i \leq n$. Consider the expression $b \sigma_{i}(a) \sigma_{i}\left(b \sigma_{i}(a)\right)$. Since $b \sigma_{i}(a) \sigma_{i}\left(b \sigma_{i}(a)\right)=$ $b \sigma_{i}(a) \sigma_{i}(b) \sigma_{i}^{2}(a)=b \sigma_{i}(a b) \sigma_{i}^{2}(a)=0$, we have $b \sigma_{i}(a)=0$ ( $B$ is $\Sigma$-rigid). We know that $B$ is reduced, which implies $0=\sigma_{i}(a) b \sigma_{i}(a) b=\left(\sigma_{i}(a) b\right)^{2}$ so $\sigma_{i}(a) b=0$. Now, since we know that $b a=0$ (from $(b a)^{2}=b a b a=0$ we have $b a=0$ ), consider the expression $a \sigma_{i}(b) \sigma_{i}\left(a \sigma_{i}(b)\right)$. Since $a \sigma_{i}(b) \sigma_{i}\left(a \sigma_{i}(b)\right)=a \sigma_{i}(b) \sigma_{i}(a) \sigma_{i}^{2}(b)=$ $a \sigma_{i}(b a) \sigma_{i}^{2}(b)=0$, then $a \sigma_{i}(b)=0$.

(ii) Again, it is enough to prove that if $a b=0$, then $a \delta_{i}(b)=\delta_{i}(a) b=0$ for every $1 \leq i \leq n$. If $a b=0,0=\delta_{i}(a b)=\sigma_{i}(a) \delta_{i}(b)+\delta_{i}(a) b$, so $-\delta_{i}(a) b=\sigma_{i}(a) \delta_{i}(b)$, whence $-\delta_{i}(a) b \sigma_{i}(a) \delta_{i}(b)=\left[\sigma_{i}(a) \delta_{i}(b)\right]^{2}$. From (i) we can see that $b \sigma_{i}(a)=0$, so $\left[\sigma_{i}(a) \delta_{i}(b)\right]^{2}=0$ and hence $\sigma_{i}(a) \delta_{i}(b)=0$ (B is reduced), so $\delta_{i}(a) b=0$. Similarly, since $b a=0$, then $\delta_{i}(b a)=\sigma_{i}(b) \delta_{i}(a)+\delta_{i}(b) a=0$, i.e., $\sigma_{i}(b) \delta_{i}(a)=-\delta_{i}(b) a$, that is, $\left(\sigma_{i}(b) \delta_{i}(a)\right)^{2}=-\delta_{i}(b) a \sigma_{i}(b) \delta_{i}(a)=0\left(a \sigma_{i}(b)=0\right.$ by (i)). Then $\sigma_{i}(b) \delta_{i}(a)=0$, which imply $\delta_{i}(b) a=0$. Hence, $a \delta_{i}(b) a \delta_{i}(b)=\left(a \delta_{i}(b)\right)^{2}=0$, i.e., $a \delta_{i}(b)=0$.

(iii) The assertion follows from (i) and (ii).

(iv) Suppose that $a \sigma^{\theta}(b)=0$ for some $\theta \in \mathbb{N}^{n}$. Then by (i) we have $\sigma^{\theta}(a b)=$ $\sigma^{\theta}(a) \sigma^{\theta}(b)=0$. Since $\sigma^{\theta}$ is injective, $a b=0$. Similarly, if $\sigma^{\theta}(a) b=0$ for some $\theta \in \mathbb{N}^{n}$, then $a b=0$.

We have the following preliminary result.

Corollary 3.4. Suppose that $A$ is a skew $P B W$ extension of a ring $R$. If $R$ is $\Sigma$-rigid and $a b=0$ for $a, b \in R$, then we obtain $a x^{\alpha} b x^{\beta}=0$ in $A$ for any $\alpha, \beta \in \mathbb{N}^{n}$.

Proof. The assertion follows from Remark 2.10 (iv).

Proposition 3.5. Let $R$ be a ring. $R$ is $\Sigma$-rigid if and only if the bijective skew $P B W$ extension $A$ is a reduced ring. In this case, $e x^{\alpha}=x^{\alpha} e$ for every $\alpha \in \mathbb{N}$ and $e=e^{2} \in R$. 
Proof. Let $R$ be $\Sigma$-rigid and suppose that $A$ is not reduced. Then there exists a non-zero element $f \in A$ such that $f^{2}=0$. Since $R$ is reduced, $f \notin R$. Following Definition 2.7, consider $f=a_{0}+a_{1} X_{1}+\cdots+a_{m} X_{m}, a_{i} \in R, 0 \leq i \leq m, a_{m} \neq 0$, with $X_{i}=x^{\alpha_{i}}=$ $x_{1}^{\alpha_{i 1}} \cdots x_{n}^{\alpha_{i n}}$, and $X_{m} \succ X_{m-1} \succ \cdots \succ X_{1}$. By Theorem 2.8 (ii) we have

$$
\begin{aligned}
f^{2} & =\left(a_{m} X_{m}+\cdots+a_{1} X_{1}+a_{0}\right)\left(a_{m} X_{m}+\cdots+a_{1} X_{1}+a_{0}\right) \\
& =a_{m} X_{m} a_{m} X_{m}+\text { other terms of order less than } X_{m} X_{m} \\
& =a_{m}\left[\sigma^{\alpha_{m}}\left(a_{m}\right) X_{m}+p_{\alpha_{m}, a_{m}}\right] X_{m}+\cdots \\
& =a_{m} \sigma^{\alpha_{m}}\left(a_{m}\right) X_{m} X_{m}+a_{m} p_{\alpha_{m}, a_{m}} X_{m}+\cdots \\
& =a_{m} \sigma^{\alpha_{m}}\left(a_{m}\right)\left[c_{\alpha_{m}, \alpha_{m}} x^{2 \alpha_{m}}+p_{\alpha_{m}, \alpha_{m}}\right]+a_{m} p_{\alpha_{m}, a_{m}} X_{m}+\cdots,
\end{aligned}
$$

where $p_{\alpha_{m}, a_{m}}=0$ or $\operatorname{deg}\left(p_{\alpha_{m}, a_{m}}\right)<\left|\alpha_{m}\right|$ if $p_{\alpha_{m}, a_{m}} \neq 0$, and $p_{\alpha_{m}, \alpha_{m}}=0$ or $\operatorname{deg}\left(p_{\alpha_{m}, \alpha_{m}}=0\right)<\left|\alpha_{m}+\alpha_{m}\right|$ if $p_{\alpha_{m}, \alpha_{m}} \neq 0$. From the equality $\operatorname{lc}\left(f^{2}\right)=$ $a_{m} \sigma^{\alpha_{m}}\left(a_{m}\right) c_{\alpha_{m}, \alpha_{m}}=0$ we obtain $a_{m} \sigma^{\alpha_{m}}\left(a_{m}\right)=0$ ( $A$ is bijective). Lemma 3.3 (iv) imply $a_{m}^{2}=0$, and so $a_{m}=0$ ( $R$ is reduced $)$, which is a contradiction. Hence, $A$ is reduced.

Conversely, since $A$ is reduced, $R$ is also reduced as a subring. Let us see that $R$ is $\Sigma$-rigid. If $a \in R$ and $a \sigma^{\alpha}(a)=0$, then $0=\sigma^{\alpha}(a) x^{\alpha} a \sigma^{\alpha}(a) x^{\alpha} a=\left(\sigma^{\alpha}(a) x^{\alpha} a\right)^{2}$, and so $\sigma^{\alpha}(a) x^{\alpha} a=0$. Thus, $0=\sigma^{\alpha}(a) x^{\alpha} a=\sigma^{\alpha}(a)\left[\sigma^{\alpha}(a) x^{\alpha}+p_{\alpha, a}\right]=\left(\sigma^{\alpha}(a)\right)^{2} x^{\alpha}+\sigma^{\alpha}(a) p_{\alpha, a}$, with $p_{\alpha, a}=0$ or $\operatorname{deg}\left(p_{\alpha, a}\right)<|\alpha|$ if $p_{\alpha, a} \neq 0$ (Theorem 2.8). Hence $\left(\sigma^{\alpha}(a)\right)^{2}=0$, that is, $\sigma^{\alpha}(a)=0$. Now, since $\sigma^{\alpha}$ is injective, we obtain $a=0$, which shows that $R$ is $\Sigma$-rigid.

Finally, let $e$ be an idempotent in $R$. Since $A$ is abelian, $e$ is central and we have the equality $e x_{i}=x_{i} e=\sigma_{i}(e) x_{i}+\delta_{i}(e)$, whence $e=\sigma_{i}(e)$ and $\delta_{i}(e)=0$. More generally, for $\alpha \in \mathbb{N}^{n}$, we can see from Proposition 2.9 that $e x^{\alpha}=x^{\alpha} e=\sigma^{\alpha}(e) x^{\alpha}+p_{\alpha, e}$, and so $e=\sigma^{\alpha}(e)$ and $p_{\alpha, e}=0$ for every element $\alpha$ of $\mathbb{N}^{n}$.

For the next proposition, suppose that the elements $c_{i, j}$ in Definition 2.1 (iv) are in the center of $R$, that is, they commute with every element of $R$.

Proposition 3.6. Suppose that $R$ is an $\Sigma$-rigid ring. Let $f=a_{0}+a_{1} X_{1}+\cdots+a_{m} X_{m}$, $g=b_{0}+b_{1} Y_{1}+\cdots+b_{t} Y_{t}$ be elements of a bijective skew $P B W$ extension $A$ of $R$. Then $f g=0$ if and only if $a_{i} b_{j}=0$ for all $0 \leq i \leq m, 0 \leq j \leq t$.

Proof. Suppose that $f g=0$. We have $f g=\left(a_{0}+a_{1} X_{1}+\cdots+a_{m} X_{m}\right)\left(b_{0}+b_{1} Y_{1}+\cdots+\right.$ $\left.b_{t} Y_{t}\right)=\sum_{k=0}^{m+t}\left(\sum_{i+j=k} a_{i} X_{i} b_{j} Y_{j}\right)$. Note that $\operatorname{lc}(f g)=a_{m} \sigma^{\alpha_{m}}\left(b_{t}\right) c_{\alpha_{m}, \beta_{t}}=0$. Since $A$ is bijective, $a_{m} \sigma^{\alpha_{m}}\left(b_{t}\right)=0$, and by Lemma 3.3 (iv), $a_{m} b_{t}=0$. The idea is to prove that $a_{p} b_{q}=0$ for $p+q \geq 0$. We proceed by induction. Suppose that $a_{p} b_{q}=0$ for $p+q=m+t, m+t-1, m+t-2, \ldots, k+1$ for some $k>0$. By Corollary 3.4 we obtain $a_{p} X_{p} b_{q} Y_{q}=0$ for these values of $p+q$. In this way we only consider the sum of the products $a_{u} X_{u} b_{v} Y_{v}$, where $u+v=k, k-1, k-2, \ldots, 0$. Fix $u$ and $v$. Consider the sum of all terms of $f g$ having exponent $\alpha_{u}+\beta_{v}$. By Proposition 2.9, Remark 2.10, and the assumption $f g=0$, we know that the sum of all coefficients of all these terms can be written as

$$
a_{u} \sigma^{\alpha_{u}}\left(b_{v}\right) c_{\alpha_{u}, \beta_{v}}+\sum_{\alpha_{u^{\prime}}+\beta_{v^{\prime}}=\alpha_{u}+\beta_{v}} a_{u^{\prime}} \sigma^{\alpha_{u^{\prime}}}\left(\sigma^{\prime} \text { s and } \delta^{\prime} \text { s evaluated in } b_{v^{\prime}}\right) c_{\alpha_{u^{\prime}}, \beta_{v^{\prime}}}=0 .
$$


By assumption we know that $a_{p} b_{q}=0$ for $p+q=m+t, m+t-1, \ldots, k+1$. So, Lemma 3.3 (iii) guarantees that the product

$$
a_{p}\left(\sigma^{\prime} \text { s and } \delta^{\prime} \text { s evaluated in } b_{q}\right) \quad\left(\text { any order of } \sigma^{\prime} s \text { and } \delta^{\prime} s\right)
$$

is equal to zero. Then $\left[\left(\sigma^{\prime} \text { s and } \delta^{\prime} \text { s evaluated in } b_{q}\right) a_{p}\right]^{2}=0$ and hence we obtain the equality $\left(\sigma^{\prime} \mathrm{s}\right.$ and $\delta^{\prime}$ s evaluated in $\left.b_{q}\right) a_{p}=0$ ( $R$ is reduced). In this way, multiplying (5) by $a_{k}$, and using the fact that the elements $c_{i, j}$ in Definition 2.1 (iv) are in the center of $R$,

$$
a_{u} \sigma^{\alpha_{u}}\left(b_{v}\right) a_{k} c_{\alpha_{u}, \beta_{v}}+\sum_{\alpha_{u^{\prime}}+\beta_{v^{\prime}}=\alpha_{u}+\beta_{v}} a_{u^{\prime}} \sigma^{\alpha_{u^{\prime}}}\left(\sigma^{\prime} \text { s and } \delta^{\prime} \text { s evaluated in } b_{v^{\prime}}\right) a_{k} c_{\alpha_{u^{\prime}}, \beta_{v^{\prime}}}=0 \text {, }
$$

whence, $a_{u} \sigma^{\alpha_{u}}\left(b_{0}\right) a_{k}=0$. Since $u+v=k$ and $v=0$, then $u=k$, so $a_{k} \sigma^{\alpha_{k}}\left(b_{0}\right) a_{k}=0$, i.e., $\left[a_{k} \sigma^{\alpha_{k}}\left(b_{0}\right)\right]^{2}=0$, from which $a_{k} \sigma^{\alpha_{k}}\left(b_{0}\right)=0$ and $a_{k} b_{0}=0$ by Lemma 3.3 (iv). Therefore, we now have to study the expression (5) for $0 \leq u \leq k-1$ and $u+v=k$. If we multiply (6) by $a_{k-1}$ we obtain

$$
a_{u} \sigma^{\alpha_{u}}\left(b_{v}\right) a_{k-1} c_{\alpha_{u}, \beta_{v}}+\sum_{\alpha_{u^{\prime}}+\beta_{v^{\prime}}=\alpha_{u}+\beta_{v}} a_{u^{\prime}} \sigma^{\alpha_{u^{\prime}}}\left(\sigma^{\prime} \text { s and } \delta^{\prime} \text { s evaluated in } b_{v^{\prime}}\right) a_{k-1} c_{\alpha_{u^{\prime}}, \beta_{v^{\prime}}}=0 .
$$

Using a similar reasoning as above, we can see that $a_{u} \sigma^{\alpha_{u}}\left(b_{1}\right) a_{k-1} c_{\alpha_{u}, \beta_{1}}=0$. Since $A$ is bijective, $a_{u} \sigma^{\alpha_{u}}\left(b_{1}\right) a_{k-1}=0$, and using the fact $u=k-1$, we have $\left[a_{k-1} \sigma^{\alpha_{k-1}}\left(b_{1}\right)\right]=0$, which imply $a_{k-1} \sigma^{\alpha_{k-1}}\left(b_{1}\right)=0$, that is, $a_{k-1} b_{1}=0$. Continuing in this way we prove that $a_{i} b_{j}=0$ for $i+j=k$. Therefore $a_{i} b_{j}=0$ for $1 \leq i \leq m$ and $1 \leq j \leq t$.

The converse follows from Corollary 3.4.

Corollary 3.7. Suppose that $R$ is an $\Sigma$-rigid ring. If $e^{2}=e \in A$, where $e=e_{0}+e_{1} X_{1}+$ $\cdots+e_{m} X_{m}$, then $e=e_{0}$.

Proof. Consider the equality $1-e=\left(1-e_{0}\right)-\sum_{i=1}^{m} e_{i} X_{i}$. By assumption $e(1-e)=0$, and then Proposition 3.6 implies $e_{0}\left(1-e_{0}\right)=0$ and $e_{i}^{2}=0$ for all $1 \leq i \leq m$. Since $R$ is reduced, $e_{i}=0$ for every $1 \leq i \leq m$, which shows that $e=e_{0}=e_{0}^{2} \in R$.

Remark 3.8. Proposition 3.6 establishes a relation between $\Sigma$-rigid rings and a skew notion of Armendariz rings. It is to be expected some stable relations between these rings and skew PBW extensions generalizing the case developed for Ore extensions of injective type presented in [19]. However, since this topic exceeds the scope and the size of this paper, in a forthcoming paper we will establish some results about this property for skew PBW extensions.

Next we prove one of the key results of this paper.

Theorem 3.9. Let $R$ be an $\Sigma$-rigid ring. Then $R$ is a Baer ring if and only if $A$ is a Baer ring.

Proof. Suppose that $R$ is a Baer ring. Let $S$ be a nonempty subset of $A$ and $S^{*}$ be the set of all coefficients of elements of $S$. Then $S^{*}$ is nonempty subset of $R$ and so $r_{R}\left(S^{*}\right)=e R$ for some idempotent $e$ of $R$. Since $e \in r_{A}(S)$, we get $e A \subseteq r_{A}(S)$. Now, 
let $0 \neq g=b_{0}+b_{1} X_{1}+\cdots+b_{m} X_{m} \in r_{A}(S)$. Then $S g=0$ and so $f g=0$ for any $f \in S$. In this way $b_{0}, b_{1}, \ldots, b_{m} \in r_{R}\left(S^{*}\right)=e R$ by Proposition 3.6. Then there exist $c_{0}, c_{1}, \ldots, c_{m} \in R$ with $g=e c_{0}+e c_{1} X_{1}+\cdots+e c_{m} X_{m}=e\left(c_{0}+c_{1} x+\cdots+c_{m} X_{m}\right) \in e A$. Hence $e A=r_{A}(S)$, that is, $A$ is Baer.

Now, assume that $A$ is Baer. Let $B$ be a nonempty subset of $R$. Then $r_{A}(B)=e A$ for some idempotent $e \in R$ by Corollary 3.7. Therefore $r_{R}(B)=r_{A}(B) \cap R=e A \cap R=e R$, which shows that $R$ is Baer.

Birkenmeier in [3], Lemma 1, establishes that if $B$ is a reduced ring, then $B$ is quasi-Baer if and only if $B$ is an abelian Baer ring. This fact together with Proposition 3.5 and Theorem 3.9 guarantee the following result about skew PBW extensions of quasi-Baer rings.

Theorem 3.10. Let $R$ be an $\Sigma$-rigid ring. Then $R$ is a quasi-Baer ring if and only if $A$ is a quasi-Baer ring.

Remark 3.11. (i) ([9], Example 2.8). Let $B=\mathbb{k}[t]$ be the polynomial ring over a field $\mathbb{k}$ and $\sigma$ be the endomorphism given by $\sigma(f(t))=f(0)$. Then $B$ is quasi-Baer but the ring $B[x ; \sigma]$ is not a quasi-Baer ring. This example shows that the assumption on $R$ (injective endomorphisms due to $\Sigma$-rigid) is not a superfluous condition in Theorem 3.10 (and, of course, Propositions 3.5 and 3.6). Another examples which show the importance of rigidness of $R$ can be found in [13], Examples 9 and 10 (1).

(ii) ([2], Example 11). There is a ring $B$ and a derivation $\delta$ of $B$ such that $B[x ; \delta]$ is a Baer ring but $B$ is not quasi-Baer. Let $B=\mathbb{Z}_{2}[t] /\left\langle t^{2}\right\rangle$, with the derivation $\delta$ such that $\delta(\bar{t})=1$ where $\bar{t}=t+\left\langle t^{2}\right\rangle$ in $B$, and $\mathbb{Z}_{2}[t]$ is the polynomial ring over the field $\mathbb{Z}_{2}$ of two elements. Consider the Ore extension $B[x ; \delta]$. If we set $e_{11}=\bar{t} x, e_{12}=\bar{t}$, $e_{21}=\bar{t} x^{2}+x$, and $e_{22}=1+\bar{t} x$ in $B[x ; \delta]$, then they form a system of matrix units in $B[x ; \delta]$. Now the centralizer of these matrix units in $B[x ; \delta]$ is $\mathbb{Z}_{2}\left[x^{2}\right]$. Therefore $B[x ; \delta] \cong M_{2}\left(\mathbb{Z}_{2}\left[x^{2}\right]\right) \cong M_{2}\left(\mathbb{Z}_{2}\right)[y]$, where $M_{2}\left(\mathbb{Z}_{2}\right)[y]$ is the polynomial ring over $M_{2}\left(\mathbb{Z}_{2}\right)$. So the ring $B[x ; \delta]$ is a Baer ring, but $B$ is not quasi-Baer.

(iii) Since prime rings are quasi-Baer, if $A$ is a bijective skew PBW extension of a prime ring $R$, then $A$ is prime ([24], Proposition 3.3 or [18], Corollary 4.2) and hence quasi-Baer.

From [13], Example 10 (2), we know that there exists a right p.p.-ring $B$ such that $B[x ; \sigma, \delta]$ is not a right p.p.-ring. This observation motives the next result for skew PBW extensions of p.p.-rings.

Theorem 3.12. Let $R$ be an $\Sigma$-rigid ring. Then $R$ is a p.p.-ring if and only if $A$ is a p.p.-ring.

Proof. Suppose that $R$ is a p.p.-ring. Let $f=a_{0}+a_{1} X_{1}+\cdots+a_{m} X_{m} \in A$. There exists an idempotent $e_{i} \in R$ with $r_{R}\left(a_{i}\right)=e_{i} R$ for $i=0,1, \ldots, m$. Let $e:=e_{0} e_{1} \cdots e_{m}$. Since every $e_{i}$ is central, $e^{2}=e$, and besides we can see that $e R=\bigcap_{i=0}^{m} r_{R}\left(a_{i}\right)$. By Proposition 3.5, we know that $f e=a_{0} e+a_{1} X_{1} e+\cdots+a_{m} X_{m} e=a_{0} e+a_{1} e X_{1}+\cdots+a_{m} e X_{m}=0$. In this way, $e A \subseteq r_{A}(f)$. Now, let $g=b_{0}+b_{1} X_{1}+\cdots+X_{t} \in r_{A}(f)$. Using the fact $f g=0$, Proposition 3.6 implies $a_{i} b_{j}=0$ for $0 \leq i \leq m$ and $0 \leq j \leq t$. Hence 
$b_{j} \in e_{0} e_{1} \cdots e_{m} R=e R$ for all $j$, which shows $g \in e A$. Therefore the equality $e A=r_{A}(f)$ is proved, that is, $A$ is a p.p.-ring.

Now, suppose that $A$ is a p.p.-ring, and consider an element $r \in R$. Then there exists an idempotent $e \in A$ with $r_{A}(r)=e A$, and by Corollary 3.7 we know that $e \in R$, so $r_{R}(r)=e R$, that is, $R$ is a p.p.-ring.

Lemma 3.1, Proposition 3.5, and Theorem 3.12 imply the following result about skew PBW extensions of p.q.-Baer rings.

Theorem 3.13. Let $R$ be an $\Sigma$-rigid ring. Then $R$ is a p.q.-Baer ring if and only if $A$ is a p.q.-Baer ring.

Remark 3.14. In Propositions 3.5 and 3.6 we do not assume that the injective endomorphisms $\sigma_{i}$ of $\Sigma$ are bijective, that is, we only use the fact that the elements $c_{i, j}$ are invertible. In this way, Theorems $3.9,3.10,3.12$, and 3.13 are valid for general skew PBW extensions satisfying these conditions on the elements $c_{i, j}$.

\section{Examples}

In this section we present some remarkable examples of skew PBW extensions which can not be expressed as Ore extensions (a more complete list can be found in [17] or [23]).

(a) Let $k$ be a commutative ring and $\mathfrak{g}$ a finite dimensional Lie algebra over $k$ with basis $\left\{x_{1}, \ldots, x_{n}\right\}$. The universal enveloping algebra of $\mathfrak{g}$, denoted $\mathcal{U}(\mathfrak{g})$, is a skew PBW extension of $\boldsymbol{k}$ (see [17]), since $x_{i} r-r x_{i}=0, x_{i} x_{j}-x_{j} x_{i}=\left[x_{i}, x_{j}\right] \in \mathfrak{g}=$ $k+k x_{1}+\cdots+k x_{n}, r \in k$, for $1 \leq i, j \leq n$. In particular, the universal enveloping algebra of a Kac-Moody Lie algebra is a skew PBW extension of a polynomial ring.

(b) The universal enveloping ring $\mathcal{U}(V, R, \mathbb{k})$ introduced by Passman [22], where $R$ is a $\mathbb{k}$-algebra, and $V$ is a $\mathbb{k}$-vector space which is also a Lie ring containing $R$ and $\mathbb{k}$ as Lie ideals with suitable relations. The enveloping $\operatorname{ring} \mathcal{U}(V, R, \mathbb{k})$ is a finite skew PBW extension of $R$ if $\operatorname{dim}_{\mathbb{k}}(V / R)$ is finite.

(c) Let $k, \mathfrak{g},\left\{x_{1}, \ldots, x_{n}\right\}$ and $\mathcal{U}(\mathfrak{g})$ be as in the previous example; let $R$ be a $k$-algebra containing $k$. The tensor product $A:=R \otimes_{k} \mathcal{U}(\mathfrak{g})$ is a skew PBW extension of $R$, and it is a particular case of crossed product $R * \mathcal{U}(\mathfrak{g})$ of $R$ by $\mathcal{U}(\mathfrak{g})$, which is a skew PBW extension of $R$ (see [20]).

(d) The twisted or smash product differential operator ring $R \#_{\sigma} \mathcal{U}(\mathfrak{g})$, where $\mathfrak{g}$ is a finite-dimensional Lie algebra acting on $R$ by derivations, and $\sigma$ is Lie 2-cocycle with values in $R$.

(e) Diffusion algebras arise in physics as a possible way to understand a large class of 1-dimensional stochastic process. A diffusion algebra (see [12]) $\mathcal{A}$ with parameters $a_{i j} \in \mathbb{C} \backslash\{0\}, 1 \leq i, j \leq n$, is an algebra over $\mathbb{C}$ generated by variables $x_{1}, \ldots, x_{n}$ subject to relations

$$
a_{i j} x_{i} x_{j}-b_{i j} x_{j} x_{i}=r_{j} x_{i}-r_{i} x_{j},
$$

whenever $i<j, b_{i j}, r_{i} \in \mathbb{C}$ for all $i<j . \mathcal{A}$ admits a $P B W$-basis of standard monomials $x_{1}^{i_{1}} \cdots x_{n}^{i_{n}}$, that is, $\mathcal{A}$ is a diffusion algebra if these standard monomials 
are a $\mathbb{C}$-vector space basis for $\mathcal{A}$. From Definition 2.1, (iii) and (iv), it is clear that the family of skew PBW extensions are more general than diffusion algebras.

Following [12], p. 22, "in the applications to physics the parameters $a_{i j}$ are strictly positive reals and the parameters $b_{i j}$ are positive reals as they are unnormalised measures of probability. We will denote $q_{i j}:=\frac{b_{i j}}{a_{i j}}$. The parameter $q_{i j}$ can be a root of unity if and only if is equal to 1 . It is therefore reasonable to assume that these parameters not to be a root of unity other than 1". If all coefficients $q_{i j}$ in (7) are nonzero, then the corresponding diffusion algebra have a PBW basis of standard monomials $x_{1}^{i_{1}} \cdots x_{n}^{i_{n}}$, and hence these algebras are skew PBW extensions. More precisely, $\mathcal{A} \cong \sigma(\mathbb{C})\left\langle x_{1}, \ldots, x_{n}\right\rangle$.

\section{Acknowledgements}

We thank the referee for his very useful remarks. Research is supported by Grant HERMES CODE 30366, Departamento de Matemáticas, Universidad Nacional de Colombia, Bogotá.

\section{References}

[1] Armendariz E.P., "A note on extensions of Baer and p.p.-rings", J. Austral. Math. Soc. 18 (1974), 470-473.

[2] Armendariz E.P., Koo H.K. and Park J.K., "Isomorphic Ore extensions", Comm. Algebra 15 (1987), No. 12, 2633-2652.

[3] Birkenmeier G.F., "Baer rings and quasicontinuous rings have a MDSN", Pacific J. Math. 97 (1981), No. 2, 283-292.

[4] Birkenmeier G.F., Kim J.Y. and Park J.K., "Principally quasi-Baer rings", Comm. Algebra 29 (2001), No. 2, 639-660.

[5] Birkenmeier G.F., Kim J.Y. and Park J.K., "Polynomial extensions of Baer and quasi-Baer rings", J. Pure Appl. Algebra 159 (2001), No. 1, 25-42.

[6] Clark W.E., "Twisted matrix units semigroup algebras", Duke Math. J. 34 (1967), 417-423.

[7] Gallego C. and Lezama O., "Gröbner bases for ideals of $\sigma$-PBW extensions", Comm. Algebra 39 (2011), No. 1, 50-75.

[8] Han J., Hirano Y. and Kim H., "Semiprime Ore extensions", Comm. Algebra 28 (2000), No. $8,3795-3801$.

[9] Han J., Hirano Y. and Kim H., "Some results on skew polynomial rings over a reduced ring", in International Symposium on Ring Theory (Kyongju, 1999), Trends Math., Birkhäuser Boston, Boston, MA (2001), 123-129.

[10] Hashemi E., Moussavi A. and Seyyed Javadi H.H., "Polynomial Ore extensions of Baer and p.p.-rings", Bull. Iranian Math. Soc. 29 (2003), No. 2, 65-86.

[11] Hashemi E. and Moussavi A., "Polynomial extensions of quasi-Baer rings", Acta Math. Hungar. 107 (2005), No. 3, 207-224. 
[12] Hinchcliffe O., "Difussion Algebras", Thesis (Ph.D.), University of Sheffield, Sheffield, 2005, $118 \mathrm{p}$.

[13] Hong C.Y., Kim N.K. and Kwak T.K., "Ore extensions of Baer and p.p.-rings", J. Pure Appl. Algebra 151 (2000), No. 3, 215-226.

[14] Hong C.Y., Kim N.K. and Lee Y., "Ore extensions of quasi-Baer rings", Comm. Algebra 37 (2009), No. 6, 2030-2039.

[15] Kaplansky I., Rings of Operators. W.A. Benjamin, Inc., New York-Amsterdam, 1968.

[16] Krempa J., "Some Examples of reduced rings", Algebra Colloq. 3 (1996), No. 4, 289-300.

[17] Lezama O. and Reyes A., "Some homological properties of skew PBW extensions", Comm. Algebra 42 (2014), No. 3, 1200-1230.

[18] Lezama O., Acosta J.P. and Reyes A., "Prime ideals of skew PBW extensions", Rev. Un. Mat. Argentina 56 (2015), No. 2, 39-55.

[19] Matczuk J., "A Characterization of $\sigma$-rigid rings", Comm. Algebra 32 (2004), No. 11, 43334336.

[20] McConnell J.C. and Robson J.C., Noncommutative Noetherian rings, Graduate Studies in Mathematics, 30, American Mathematical Society, Providence, RI, 2001.

[21] Nasr-Isfahani A.R. and Moussavi A., "Baer and quasi-Baer differential polynomial rings", Comm. Algebra 36 (2008), No. 9, 3533-3542.

[22] Passman D.S., "Prime ideals in enveloping rings", Trans. Amer. Math. Soc. 302 (1987), No. $2,535-560$.

[23] Reyes A., "Ring and module theoretical properties of skew PBW extensions", Thesis (Ph.D.), Universidad Nacional de Colombia, Bogotá, 2013, 142 p.

[24] Reyes A., "Jacobson's conjecture and skew PBW extensions", Rev. Integr. Temas Mat. 32 (2014), No. 2, 139-152. 